

\title{
Study of the depletion depth in a frontside biased CMOS pixel sensors
}

\author{
J. Heymes, ${ }^{1}$ A. Dorokhov, M. Kachel, and J. Baudot \\ Université de Strasbourg, CNRS, IPHC UMR 7178, \\ F-67000 Strasbourg, France \\ E-mail: julian.heymes@iphc.cnrs.fr
}

Aвstract: Depletion of the sensitive volume for semiconductor based detectors is a key to achieve high performance. It is for instance required for charged particle detection in highly radiative environment and for X-ray spectroscopy.

PIPPER-2 is a CMOS pixel sensor featuring an architecture that allows the application of the reverse bias of the pn junction from the frontside (cathode), on the electronic side, without process modification. Biasing voltages up to $45 \mathrm{~V}$ have been applied to sensor prototypes fabricated on two different high resistivity substrates: a thin epitaxial layer $(1 \mathrm{k} \Omega \mathrm{cm})$ and a $40 \mu \mathrm{m}$ thick bulk substrate $(600 \Omega \mathrm{cm})$.

Calculations from a simplified analytical model and 3D-TCAD simulations were conducted to predict the evolution of the depletion depth with the bias voltage. These expectations were compared to measurements of PIPPER-2 illuminated with two X-ray energies.

We conclude that the frontside biasing method allows the full-depletion of the thin epitaxial layer. In contrast, depletion of the bulk substrate reaches about half-depth but X-rays are still detected over the full depth .

\footnotetext{
${ }^{1}$ Corresponding author.
} 


\section{Contents}

1 Introduction 1

2 Frontside biasing depletion $\quad 2$

2.1 Frontside biasing for CPS depletion 2

2.2 The PIPPER-2 sensor 2

3 Depletion depth expectations from first principle 3

3.1 Analytical approximations with point-like diodes 3

3.2 Finite element simulations 5

4 Experimental estimates of the depletion depth $\quad 8$

$\begin{array}{llr}4.1 & \text { Method based on count ratio } & 9\end{array}$

4.2 Evaluation from counts in full-energy peaks $\quad 11$

$\begin{array}{ll}4.3 & \text { Evaluation from all of the hits } \\ & 12\end{array}$

5 Conclusions and outlooks $\quad 13$

$\begin{array}{lll}\text { A Analytical model } & 14\end{array}$

$\begin{array}{lll}\text { A.1 Hemispherical depleted volume from a small diode } & 14\end{array}$

$\begin{array}{lll}\text { A.2 Approximation for merged depleted volumes } & 15\end{array}$

\section{Introduction}

CMOS pixel sensors (CPS) are now well established as silicon-based devices for a number of scientific applications [1-4]. However, when optimal detection requires a depleted sensitive volume exceeding typically $20 \mu \mathrm{m}$, most CPS will experience severe limitations because their depletion depth reaches only a few micrometers.

Like other semiconductor detectors, CPS require some level of depletion to operate properly. But until recent years, most CPS featured only a depletion depth of a few micrometers, much thinner than the whole depth of the sensitive volume. Under this configuration the charge generated by the radiation to be detected, is collected through a combination of thermal motion and drift. In order to both increase the depth of the sensitive volume and decrease the charge collection time, various recent developments [5-7] target to reach larger or even full depletion of the sensitive volume. Such a new generation of sensors are appealing to a wide range of applications, from High Energy Physics to non-destructive-tests with electrons or soft X-rays.

Depletion in semiconductors relies on two basic ingredients: substrate resistivity $\rho$ and the reverse bias voltage $V_{R B}$. The depletion depth follows a law of the form $\left(\rho V_{R B}\right)^{\gamma}$, with $\gamma<1$ depending on the geometric configuration of the electric potential. Solutions to increase depletion 
choose a high resistivity and/or a high voltage. When implemented into the CMOS technology, usually a low voltage and low resistivity substrate process are used. These recipes necessarily impact the fabrication process or the electronic design. Moreover some post-processing steps might be required.

In this work, we propose to exploit the availability of high resistivity substrate in a CMOS process. The biasing voltage is applied from the cathode side of the collecting diode through the collecting diode and require a dedicated pixel design. This solution should allow for relatively small pixel sizes, typically $20 \mu \mathrm{m}$, and targets low noise performance.

We intend to apply the frontside biasing method to deplete various types of substrates depending on the application. For charged particle tracking, thin epitaxial layers offer an excellent compromise between generating a high enough signal and limiting the overall thickness of the detector. Turning to X-ray detection, bulk substrates are a priori a better choice since thickness is beneficial for quantum efficiency.

The key question addressed in this paper concerns the shape and the depth of the depleted volume, enabled by the proposed frontside biasing technique. The next section describes the concept of frontside biasing with small diodes and its implementation in the PIPPER-2 prototype. In section three, we derive expectations for the depletion depth from first principles, both with a simplified analytic model and finite element calculus. Measurements with soft X-rays are reported in section four and are used to derive an experimental estimate of the depleted depth.

\section{Frontside biasing depletion}

\subsection{Frontside biasing for CPS depletion}

The proposed method consists of applying the bias voltage to the in-pixel collecting diodes implemented in the top side of the sensitive volume, which is expected to feature the highest resistivity. Since usually collecting nodes are DC-coupled to the in-pixel amplifiers, their working point as well as the breakdown voltage of the technology limits the maximum applicable bias voltage.

To allow higher bias voltages and hence deeper depletion, the proposed frontside biasing requires AC-coupling between the collecting node and the amplifier part of the pixel. The collection node is biased through a forward biased diode. Figure 1 depicts the electrical schematic of a simple pixel following this architecture. While low DC voltages $\left(\mathrm{V}_{\text {bias }}\right.$, vdda) bias the readout electronics, a higher voltage (HV) can be applied to the collecting node.

In addition to the depletion, the frontside biasing scheme brings various benefits. First neither process nor design rules modifications are required. Then, different types of substrates can be used, provided their resistivity is high enough, typically close or beyond $1 \mathrm{k} \Omega \mathrm{cm}$. With substrates featuring a thin epitaxial layer (below $40 \mu \mathrm{m}$ ), the full sensitive depth is expected to be depleted. In contrast, low doped and thick bulk substrates will probably be only partially depleted. The pixel design needs only a small change, which consists of introducing the coupling capacitor.

\subsection{The PIPPER-2 sensor}

PIPPER-2 (Pixelated sensor for Ionizing Particles and Photons Energy Resolved detection) is a prototype CMOS pixel sensor implementing the frontside biasing technique introduced previously. 


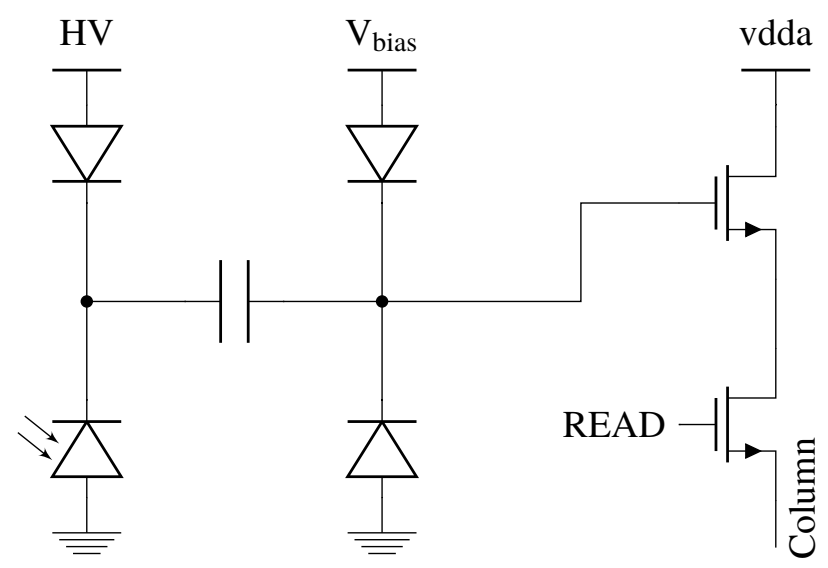

Figure 1. Schematic of the PIPPER-2 pixel. The substrate is biased from the top through a forward bias diode and the collecting diode. The collection node is AC-coupled to a source follower. A set of diodes after the coupling at the input of the amplifier is used to set the DC point.

It was designed and fabricated in $180 \mathrm{~nm}$ CMOS Image Sensor process. The prototypes were produced on two different substrates: an $18 \mu \mathrm{m}$ high resistivity $(>1 \mathrm{k} \Omega \mathrm{cm}$ ) P-type epitaxial layer dubbed HR18 in the following, and a high resistivity $(>600 \Omega \mathrm{cm})$ P-type Czochralski bulk substrate, noted CZ, thinned to $280 \mu \mathrm{m}$ at the foundry. A few CZ-bulk sensors have then been thinned to $50 \mu \mathrm{m}$. Taking into account the $10 \mu \mathrm{m}$ thick metalisation layers of the CMOS process, those sensors feature consequently a potential sensitive volume of $\approx 40 \mu \mathrm{m}$. The backside of these thinned sensors is passivated through the PULSION ${ }^{\circledR}$ process, allowing to keep a thin entrance window ( $\left.\approx 20-50 \mathrm{~nm}\right)$ [8].

The PIPPER-2 prototype is composed of four submatrices, each organized in 8 columns and 128 rows of square pixels with $22 \mu \mathrm{m}$ pitch. The submatrix selected for acquisition is read out using rolling-shutter, where each column is independently connected to an output of the sensor. Three submatrices are designed to explore collection performance with various collecting diode configuration while the fourth submatrix embeds an amplifier design. Each diode is AC-coupled to a source follower using a fringe capacitor which has been preferred over MIM capacitors because of lower leakage current, and higher breakdown voltage. The DC point after the coupling is set using a set of diodes that are isolated from the sensitive volume with deep P-well.

A schematic of the pixel is shown in figure 1. The further studies will focus on the submatrix which features a collecting diode of $5 \mu \mathrm{m}$ and coupling capacitor of $21 \mathrm{fF}$.

\section{Depletion depth expectations from first principle}

\subsection{Analytical approximations with point-like diodes}

The width of the depleted region of the 1D-model of a pn junction under reverse biasing can be derived from Poisson equation and electric neutrality [9]:

$$
W_{R B}=\sqrt{\frac{2 \epsilon_{S i}}{q}\left(\frac{N_{A}+N_{D}}{N_{A} N_{D}}\right)\left(V_{b i}+|V|\right)},
$$


with $\epsilon_{S i}$ being the permittivity of silicon, $q$ the elementary charge, $N_{D}$ and $N_{A}$ the donors and acceptors densities, $V_{b i}$ the built-in voltage, and $V$ the reverse bias. Standard silicon detectors exploit an asymetric pn junction, for instance with a highly doped $\mathrm{N}$-side $\left(N_{D} \gg N_{A}\right)$. In this case the depleted area extent mostly in the P-side and the expression of its width simplifies to:

$$
W_{R B} \approx W_{P}=\sqrt{\frac{2 \epsilon_{S i}}{q} \frac{1}{N_{A}}\left(V_{b i}+|V|\right)} .
$$

The previous equations assume an infinite (1D-model) junction area. The proposed frontside biasing architecture obviously departs strongly from this configuration. As a matter of fact, with a diameter of a few micrometers, the junction covers a marginal part of the total pixel area. For PIPPER-2, the circular-shape collecting diode features a diameter of $5 \mu \mathrm{m}$ and account only for $4 \%$ of the total pixel area ( $22 \mu \mathrm{m}$ pitch).

In order to approximate analytically a solution of the Poisson equation for this small diode geometry, one should assume a 3-D shape for the equi-potential lines on both sides of the junction. Still considering the asymmetric case with highly doped $\mathrm{N}$-side $\left(N_{D} \gg N_{A}\right)$, the interesting shape is of course the depleted volume within the P-side. For instance, J. Lincelles considered a 3-dimensional ellipsoid for a study limited to low biasing values [10]. Here we choose simply a hemispheric volume. Calculus are detailed in appendix A and yields the following expression for the radius of the hemispherical depleted area:

$$
W_{P}=\sqrt[3]{\frac{3}{2 \pi}\left(\sqrt{\frac{2 \epsilon_{S i}}{q N_{A}} \mathcal{A}^{2}\left(V_{b i}+|V|\right)}+\mathcal{V}_{\text {diode }}\right)},
$$

where $\mathcal{A}$ is the junction area and the other parameters are unchanged from equation 3.2.

However, the obtained expression does not take into account the finite area of the pixel, defined by the pitch $s$. When the width of the depleted volume becomes greater than half the pitch $\left(W_{P}>s / 2\right)$, the depleted zone of the pixel merges with the ones from the four adjacent pixels. Similarly, when the width becomes greater than half the diagonal of the pixel area $\left(W_{P}>\sqrt{p^{2} / 2}\right)$, further connections are done with the four additional pixels at the edges.

When depleted volumes of neighbouring pixels start to merge, these individuals volumes can no longer be consider as plain hemispheres but rather as truncated hemispheres with caps cut out. To match electric neutrality, the depleted volumes should then exceed the one of the truncated hemisphere. This phenomena extents the depleted depth by a quantity $W_{\text {merging }}$, which depends on the volume merged.

The latter is computed in appendix A and the final depleted width expression is then:

$$
W_{P_{t o t}}=W_{P}+\frac{\frac{2}{3} \pi\left(W_{P}-\frac{s}{2}\right)^{2}\left(2 W_{P}+\frac{s}{2}\right)}{s^{2}} .
$$

We stress that the two expressions 3.3 and 3.4 derived here bear strong simplifications and do not correspond to a proper solution of electromagnetic equations. However, we expect them to provide some indications on the depletion depth evolution with the bias voltage. Figure 2 compares the three equations approximating the evolution of the depleted width with the bias voltage. The comparison 


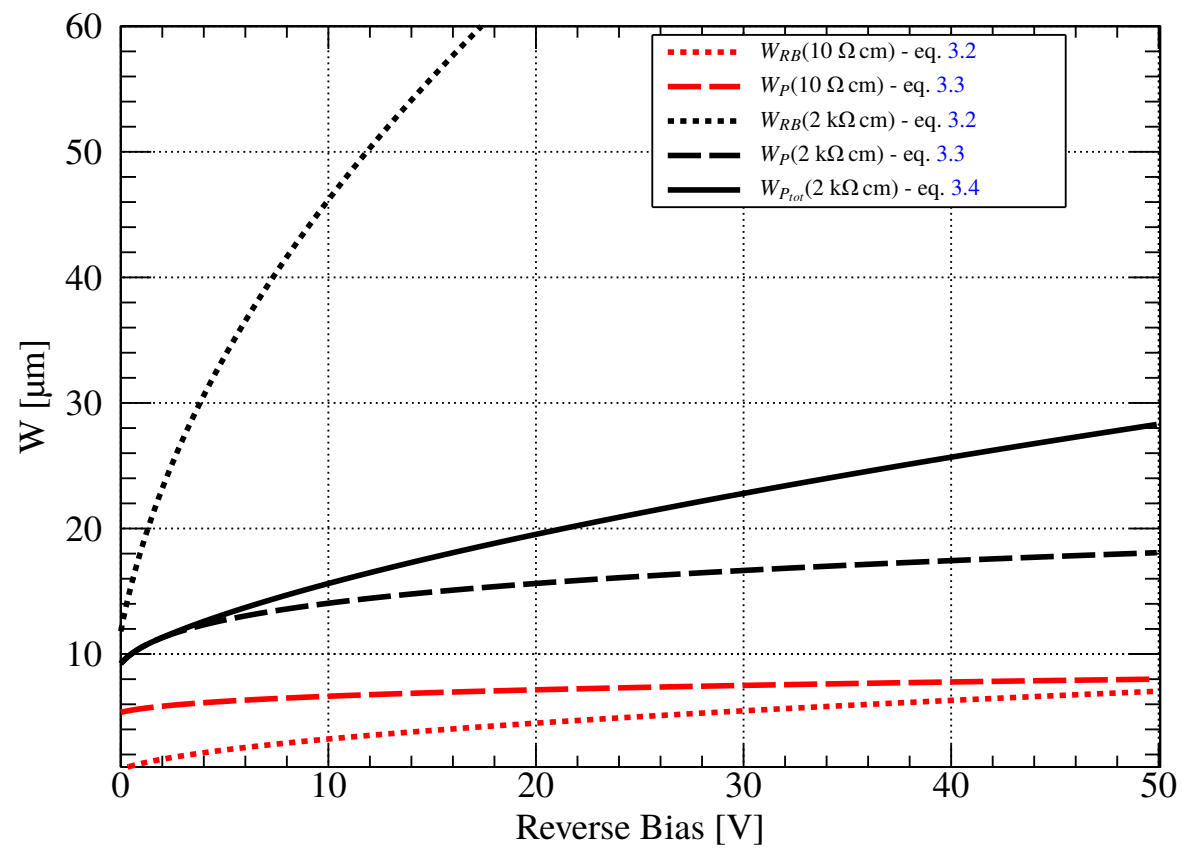

Figure 2. Analytical estimates of the depleted width in a reverse biased pn junction for two geometries and two substrate resistivities. Red color corresponds to $10 \Omega \mathrm{cm}$ and black color to $2 \mathrm{k} \Omega \mathrm{cm}$. The dotted lines are obtained with the 1D junction model of equation 3.2, while the dashed lines follow the point-like model without volume merging of equation 3.3. The solid line still corresponds to the point-like junction but, in addition, accounts for the merging of the depleted volumes from adjacent pixels depicted by equation 3.4.

is done with two resistivities for the P-type substrate: $10 \Omega \mathrm{cm}$ corresponding to the low-resistivity standard of CMOS substrate and $2 \mathrm{k} \Omega \mathrm{cm}$ standing for a high-resistivity material. For the lowest resistivity substrate, the approximated depth stays under the modest value of $10 \mu \mathrm{m}$ irrespective of the configuration modeled, the 1D-infinite or the 3D point-like junction. As expected, the low resistivity simply does not enable sizeable depletion within the bias voltage range considered.

With a high resistivity substrate, the results contrast strongly. The infinite junction model of equation 3.2 predicts overly optimistic depleted widths beyond $50 \mu \mathrm{m}$ already for $10 \mathrm{~V}$. On the contrary, the point-like junction approximation of equations 3.3 and 3.4 display much slower evolution with the bias voltage. At $50 \mathrm{~V}$, the depleted width is predicted to reach only $28 \mu \mathrm{m}$ even when taking into account the merging of the depleted volumes from adjacent pixels.

\subsection{Finite element simulations}

Although the previously discussed point-like diode analytical approximations introduced pixel geometries considerations, such as small junction area and finite pixel size, it does not take into account real features such as doping profiles and deep P-wells structures insulating CMOS transistors from the substrate. To explore the influence of these parameters and cross-check the validity of the analytical approximations, finite elements simulations with TCAD tools ${ }^{1}$ are conducted.

\footnotetext{
${ }^{1}$ https://www.synopsys.com/silicon/tcad.html
} 
The 3D geometry implemented sticks as close as possible to the pixel present in the PIPPER2 sensor. The charge collecting node is made of a centered $5 \mu \mathrm{m}$ diameter round $\mathrm{N}$-well with a doping profile extracted from the foundry documentation. This diode sits directly in the P-sensitive volume. It is surrounded by deep P-wells, whose doping profile is also from the foundry documentation, in which CMOS micro-circuits are implemented for insulation with the detection volume. The forward bias diode composed of a $\mathrm{P}++$ implant placed in the $\mathrm{N}$-well through the oxide with the bias contact is implemented. A circular P++ implant also sits in the deep P-well structure through the oxide with the ground contact.

The two types of substrates used for PIPPER-2 are reproduced. The epitaxial layer (HR18 model) is implemented according to the doping profile measured through spreading resistance profiling (SRP) over $25 \mu \mathrm{m}$. For the bulk substrate ( $\mathrm{CZ}$ model), constant doping profiles are used. Four different resistivities are considered: $600 \Omega \mathrm{cm}$ (minimum guaranteed by the manufacturer of the wafer), $1 \mathrm{k} \Omega \mathrm{cm}, 2 \mathrm{k} \Omega \mathrm{cm}$, and $>10 \mathrm{k} \Omega \mathrm{cm}$ (exact value of the maximum of the HR18 doping profile). The total thickness of the $\mathrm{CZ}$ model is $60 \mu \mathrm{m}$.

For limiting the computation time while still allowing for fine meshing, the pixel symmetry is exploited. The 3D pixel structure is simulated over the full substrate depth but only over a quarter of the pixel surface. Computations treat interfaces as mirrors, which results in same outcomes as for an infinite matrix of full pixels.

A single quasi-stationary simulation is carried out for each structure (one HR18 and four CZ) where the biasing voltage is progressively increased from $0 \mathrm{~V}$ to $45 \mathrm{~V}$. Post-simulation analysis allows study of the electrical properties relevant for the charge transfer. 2D maps of the hole density and the electric field, obtained for three bias voltages, are presented in figure 3 for the $\mathrm{CZ}$ substrate at $600 \Omega \mathrm{cm}$ and the HR18 substrate.

The thin white contour corresponds to the limit of the depleted volume as computed by TCAD, based on a lower limit of the hole density. Figure 4 displays the maximal depleted depth obtained from TCAD calculus as well as the expectation from the point-like analytical model from equation 3.4.

Focusing first on the maximal depth of the depleted volume in the CZ bulk substrate, it appears that the analytical approximations (dashed and dotted lines in figure 4) and the TCAD calculus (solid lines in figure 4) agree rather well on the depth slope with the bias voltage. This agreement tend to confirm the assumption that merging of the depleted volumes from neighbouring pixels plays an important role.

However, the two predictions do not match below a bias voltage of $10 \mathrm{~V}$. In addition, if the expectations do not differ for the resistivity of $600 \Omega \mathrm{cm}$, for the $2 \mathrm{k} \Omega \mathrm{cm}$ resistivity analytical prediction exceed the TCAD one. The difference reaches $25 \%$ at $40 \mathrm{~V}$ biasing.

An explanation for the over-optimistic prediction from the analytical model can be found in the shape of the depleted volume. Figure 3 clearly shows that the depleted contour quickly differs from the hemisphere assumed by the analytical approximation. The TCAD computations indicate that the depleted volume is rather shaped like a light bulb due to the constraint of the grounded P-wells surrounding the point-like pn junction. This very constraint generates also the behaviour at very 


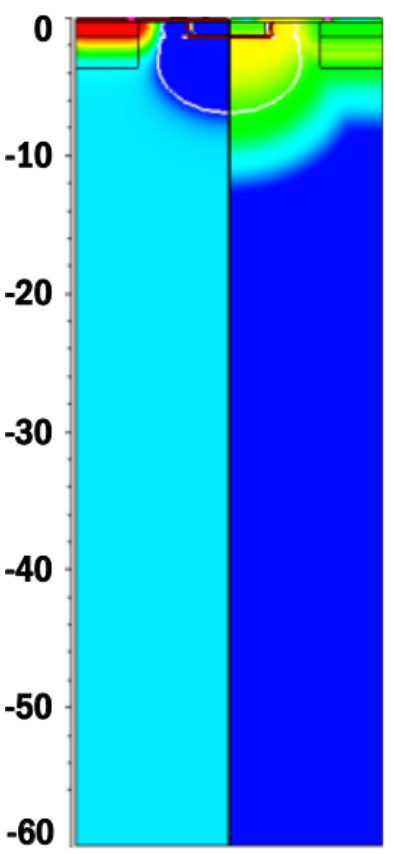

(a) $2 \mathrm{~V}$

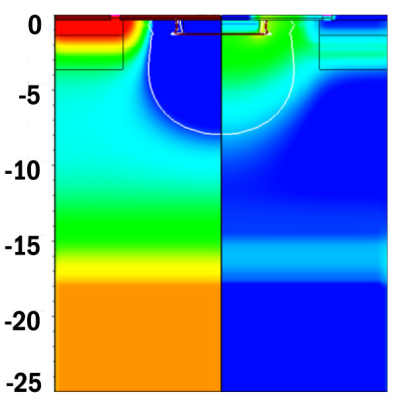

(e) $2 \mathrm{~V}$

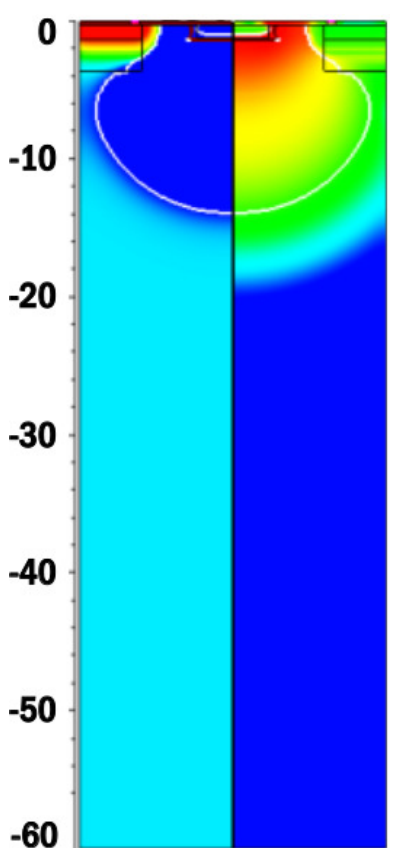

(b) $20 \mathrm{~V}$

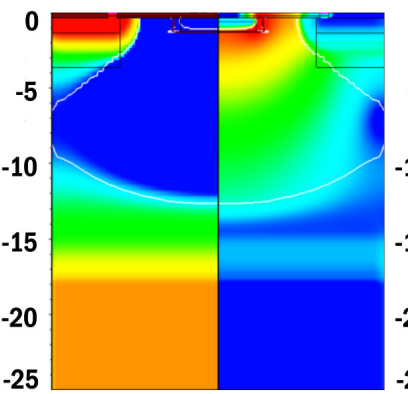

(f) $20 \mathrm{~V}$
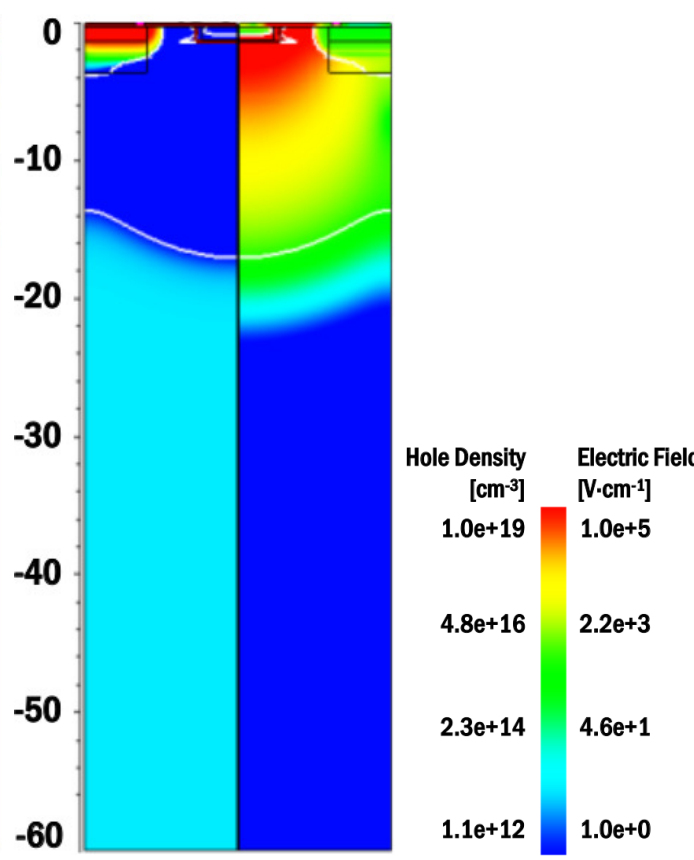

(c) $45 \mathrm{~V}$

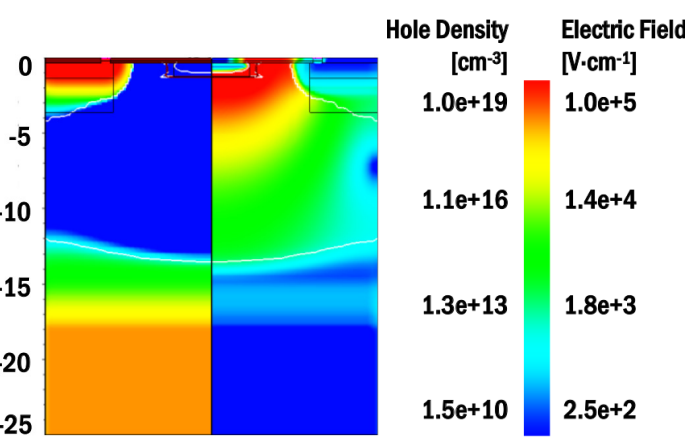

(g) $45 \mathrm{~V}$

Figure 3. Behaviour of the depletion depth with the $\mathrm{CZ}$ model using a $60 \mu \mathrm{m}$ thick $600 \Omega \mathrm{cm}$ bulk substrate $(\mathrm{a}-\mathrm{c})$ and the thin epitaxial layer model with the realistic doping profile $(\mathrm{e}-\mathrm{g})$. The biasing voltages applied are $(\mathrm{a}, \mathrm{e}) 2 \mathrm{~V},(\mathrm{~b}, \mathrm{f}) 20 \mathrm{~V}$ and $(\mathrm{c}, \mathrm{g}) 45 \mathrm{~V}$. On the left side part of each cross section, the dark blue area is where the density of holes is below $5 \%$ of the acceptors concentration. On the right part the corresponding electric field is shown.

high resistivity, above $10 \mathrm{k} \Omega \mathrm{cm}$, for which the depleted depth clearly saturates after a rapid growth up to about $10 \mathrm{~V}$ biasing.

As a conclusion for the PIPPER-2 pixel implemented in a bulk substrate, we expect from TCAD computations that $40 \mathrm{~V}$ bias will deplete $17-18 \mu \mathrm{m}$ for resistivities in the range $0.6-1 \mathrm{k} \Omega \mathrm{cm}$. Increasing the resistivity to $2 \mathrm{k} \Omega \mathrm{cm}$ deplete about 5 more micrometers.

The situation differs substantially with the epitaxial layer (HR18 model), where the rising doping gradient at $18 \mu \mathrm{m}$ depth already stops the growth of the depletion, which only steps up from 


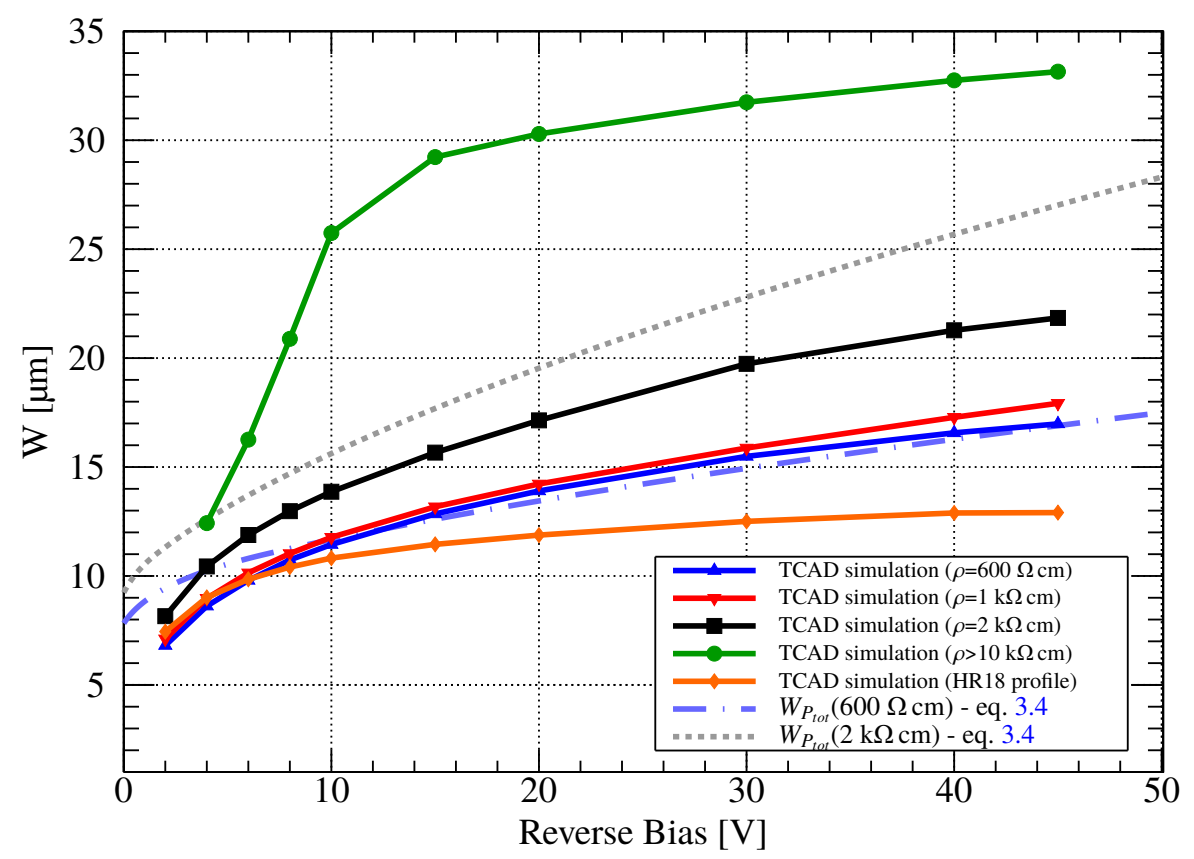

Figure 4. TCAD simulated depletion depth reached in a bulk substrate of various resistivities: $600 \Omega \mathrm{cm}$, $1 \mathrm{k} \Omega \mathrm{cm}, 2 \mathrm{k} \Omega \mathrm{cm}$, and $>10 \mathrm{k} \Omega \mathrm{cm}$ and in the HR18 structure. The simulations are compared to the point like diode equation (eq. 3.4) for a bulk resistivity of $600 \Omega \mathrm{cm}$, and of $2 \mathrm{k} \Omega \mathrm{cm}$

12 to $13 \mu \mathrm{m}$ when the biasing voltage increase from 20 to $40 \mathrm{~V}$.

It is interesting to observe that the electric field, shown on the right part of figure 3, fades away a few micrometers after the maximal depletion extension. Figure 5 provides a more accurate evolution of the electric field with depth.

In the HR18 substrate case (figure 5), the field strength stays actually almost constant from 13 to $17 \mu \mathrm{m}$, suggesting that charge collection could extend beyond the depletion volume by a maximum of $4 \mu \mathrm{m}$. Nevertheless, the doping, and hence hole concentration, rises steeply in this region and trapping could also prevent any significant amount of charge to actually reach the depleted volume. Such extension of non-zero electric field appears as well for the bulk CZ substrate (figure $5 b$ ). In this case, the field strength falls down exponentially with depth beyond the maximal depleted depth, extending about $10 \mu \mathrm{m}$ away. Dedicated charge transport simulations would be however required to make any quantitative predictions about charge collection.

\section{Experimental estimates of the depletion depth}

In the following, we base our experimental estimates of depleted depths enabled by the front-side biasing approach on detection by PIPPER-2 sensors of monochromatic X-rays available in our laboratory. However these source rates are not precisely known. Consequently we derive our results from count ratios and a reference thickness taken from TCAD expectations, as explained below. 


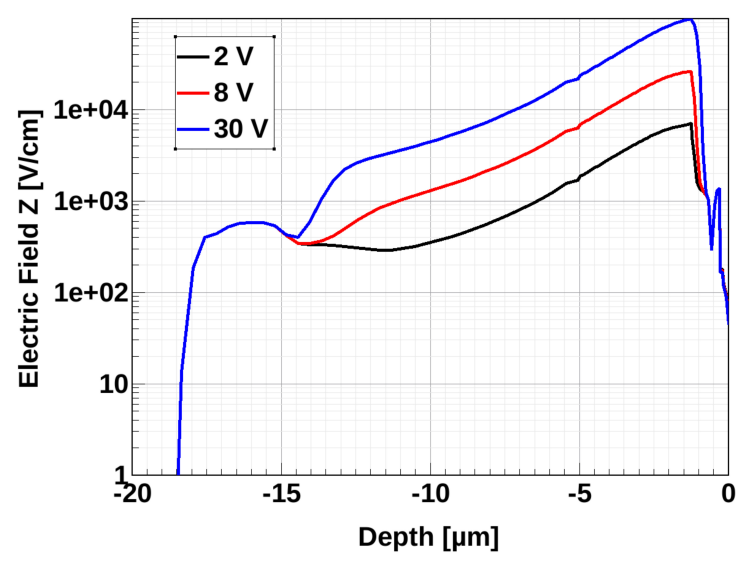

(a)

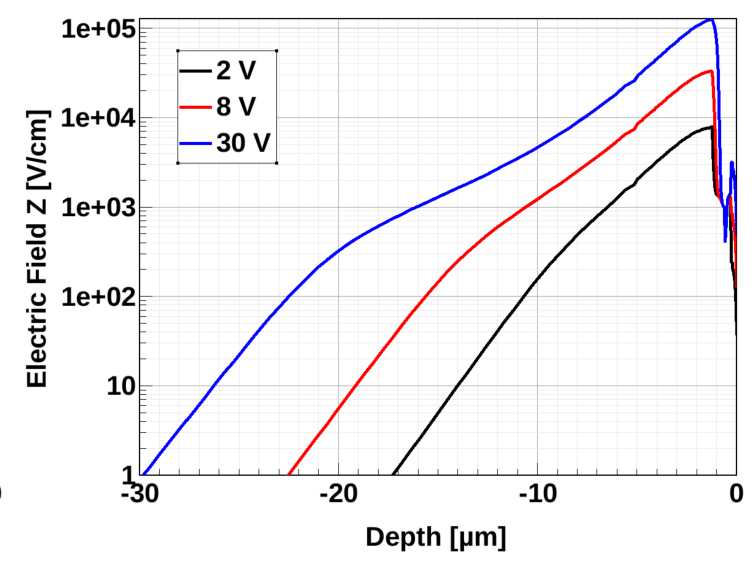

(b)

Figure 5. Simulations of the electric field along the Z-axis for different diode bias voltage ( $2 \mathrm{~V}, 8 \mathrm{~V}$, and $30 \mathrm{~V}$ ) in the (a) HR18 and (b) $\mathrm{CZ}$ with a $2 \mathrm{k} \Omega \mathrm{cm}$ structures

\subsection{Method based on count ratio}

Attenuation of X-Rays in a given material follows the well-known exponential Beer-Lamber law:

$$
I(z)=I(0) e^{-z \mu(E) \rho},
$$

where $I(z)$ is the photon intensity at depth $z, \rho$ the material density and $\mu(E)$ the attenuation power, which depends only on the photon energy $E$ and is well tabulated. The number of counts $N$ observed in a detector over a sensitive thickness $z$ is then proportional to $1-I(z)$. Considering two different sensitive thicknesses, $z_{0}$ and $z_{1}$, but identical initial intensity and for a fixed photon energy $E$, the ratio $R$ of observed counts is given by:

$$
R_{01}=\frac{N_{1}}{N_{0}}=\frac{1-e^{-z_{1} \mu(E) \rho}}{1-e^{-z_{0} \mu(E) \rho}} .
$$

Additionally, equation 4.2 assumes that the sensor ability to detect X-rays does not depend on the depth of interaction.

If depth $z_{0}$ is known and considered as a reference depth, the depth $z_{1}$ can then be inferred from the formula:

$$
z_{1}=-\frac{\log \left(R e^{-z_{0} \mu(E) \rho}-R+1\right)}{\mu(E) \rho} .
$$

In the following, we have chosen for reference the measurements ( $N_{0}$ counts) obtained with PIPPER-2 fabricated on the epitaxial layer substrate (HR18) and biased at $30 \mathrm{~V}$. In section 4, we have inferred from TCAD simulations that the depleted depth in this case is $z_{0}=13 \mu \mathrm{m}$.

The other parameters required to exploit equation 4.3 are the silicon density, taken as $\rho=$ $2.33 \mathrm{~g} \mathrm{~cm}^{-3}$, and the attenuation lengths for the two energies tested: $\mu(5.89 \mathrm{keV})=154.82 \mathrm{~cm}^{2} \mathrm{~g}^{-1}$ and $\mu(17.48 \mathrm{keV})=6.17 \mathrm{~cm}^{2} \mathrm{~g}^{-1}$ taken from [11]. 


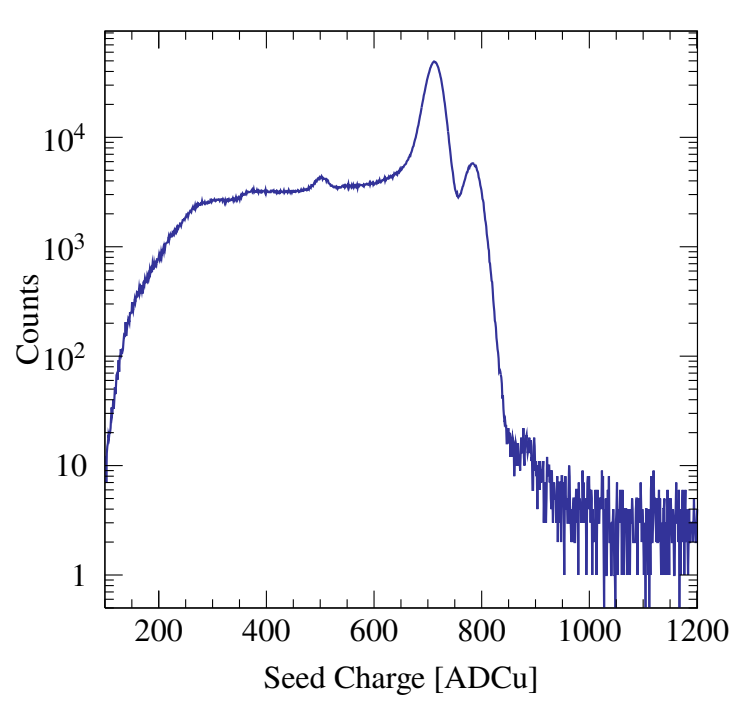

(a) ${ }^{55} \mathrm{Fe}$

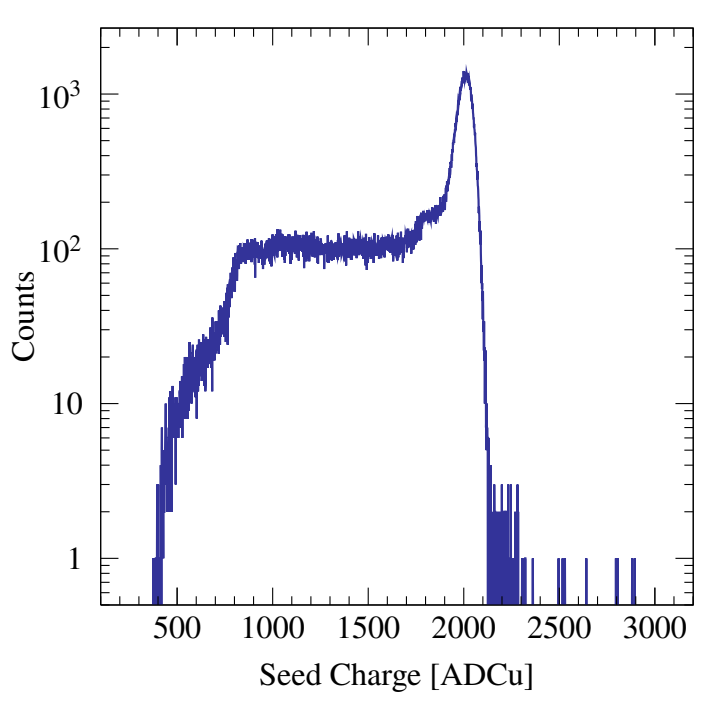

(b) Mo

Figure 6. Example of distribution of the signal observed on the seed pixel for selected hits (see text). These data were taken with PIPPER-2 fabricated on the epitaxial layer (HR18), biased at $30 \mathrm{~V}$, and illuminated with a ${ }^{55} \mathrm{Fe}$ radioactive source (a) and with X-Rays generated with a molybdenum cathode (b).

PIPPER-2 sensors, featuring the thin epitaxial layer and the thinned bulk substrate, have been front-side illuminated with photons of different energies: $5.89 \mathrm{keV}$ from a sealed ${ }^{55} \mathrm{Fe}$ source and $17.48 \mathrm{keV}$ from a Rigaku D/MAX-B X-ray generator (with a graphite monochromator in the sample chamber). In both cases, the photon rate was sufficiently low with respect to the sensor integration time (about $40 \mu \mathrm{s}$ ) to limit possible overlap of X-ray impacts on the pixel matrix. Particular care was taken of the sensor-source position in order to ensure that the rate is equivalent for irradiation on various sensors.

The analogue output of each pixel was digitized with an external 14-bits analogue to digital converter. For each bias voltage applied, 3000000 frames have been acquired followed by a standard clustering analysis to individualise X-ray impacts. Hits were selected by requiring that the sum of the signals from the pixels inside the cluster exceed 250 ADC units for the $5.89 \mathrm{keV}$ energy and $800 \mathrm{ADC}$ units for the $17.48 \mathrm{keV}$ energy. The distribution of the signal observed on the seed pixel (the one with highest signal in the cluster) for the low energy X-rays is displayed in figure 6.

The distribution reflects the two kinds of detections occurring when X-rays impact the sensor. The clear peak on the right part corresponds to situation where the charge generated by the photon is detected in a single pixel, the so-called full-energy peak. We expect that such detection happens in the depleted volume of the sensor, where the drift electric field is strong.

The flat tail extending from the full-energy peak toward low values is due to detection where only part of the charge is collected on the seed pixel. This situation mostly ${ }^{2}$ arises for photo-conversions

${ }^{2}$ Other processes could lead to overall missed charge, but are not dominating. For instance the silicon escape peak is visible in figure 6. 


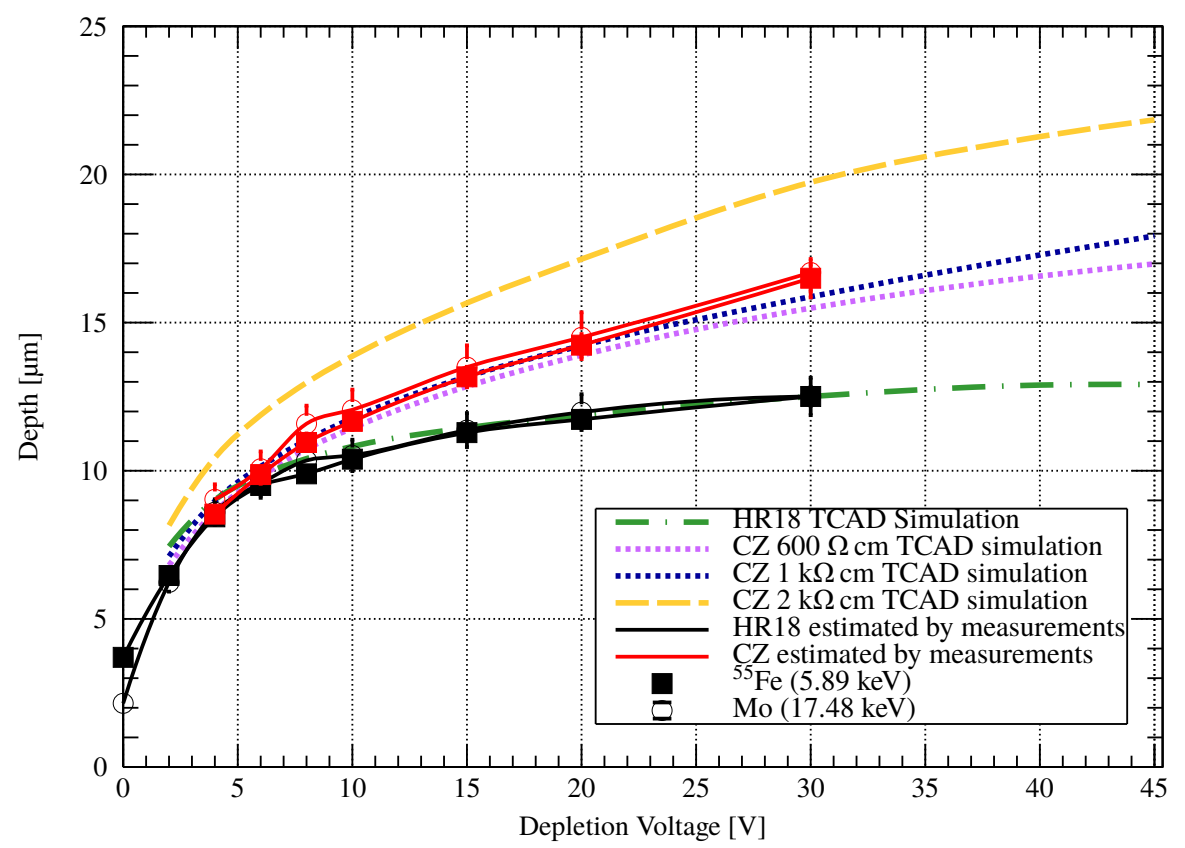

Figure 7. Estimated depletion depth for the two substrates (HR18 and CZ) used for PIPPER-2, based on the count ratio method for hits selected in the full-energy peak (solide lines). Dotted and dashed lines correspond to various expectations from TCAD simulations.

either at the pixel-edges either at the bottom of the sensitive volume, where diffusion significantly spreads charge in the lateral plane (transverse to the depth direction). Part of this events could still happen within the depleted volume (especially those at the pixel edges) but could also originate from the non-depleted but still sensitive volume.

Consequently, we count the X-ray detected in two ways. The first one restricts the number of counts to those in the full-energy peak. And the second one keeps the total number of counts in the distribution. The next two sections discuss the evaluation of the depleted depth with both count estimates.

\subsection{Evaluation from counts in full-energy peaks}

A fit of the calibration peak provides the peak position $(\mu)$ and its standard deviation $(\sigma)$. Integrating the entries under the calibration peak $(\mu \pm 3 \sigma)$ allows the selection of a majority hits whose charge has been generated within the depleted volume (or at its close boundary) and collected on one pixel by the electric field.

A fit of the full-energy peak provides the peak position $(\mu)$ and its standard deviation $(\sigma)$. Integrating the number of entries within $(\mu \pm 3 \sigma)$ intends to select hits generated within the depleted volume.

The estimated depths for both substrates, epitaxial layer (HR18) and bulk (CZ), are then based on equation 4.3. Results are displayed in figure 7 with expectation from the TCAD simulations 


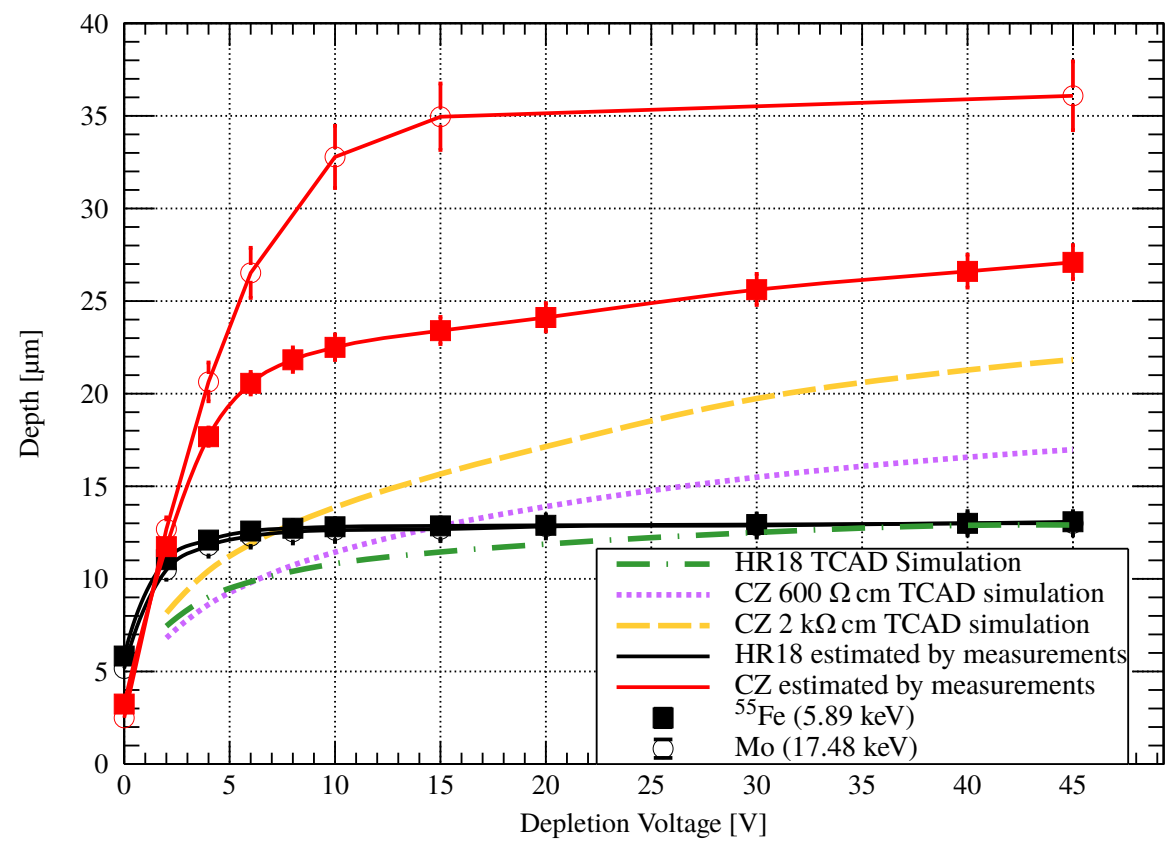

Figure 8. Estimated depth reached for good collection performance, using ratios of the total amount of entries in the seed pixel charge spectrum for selected hits (above a minimum charge must be deposited) with a TCAD simulation result as a reference point: EPI $18 \mu \mathrm{m}$ at $40 \mathrm{~V}$ evaluated at $13 \mu \mathrm{m}$.

superimposed. Starting from $2 \mathrm{~V}$ and within $1 \mu \mathrm{m}$, the epitaxial (HR18) layer behavior matches the prediction, which gives confidence in our choosing this configuration for reference.

The experimental evaluation of the depletion depth in the bulk substrate $(\mathrm{CZ})$ is closer to the TCAD expectation for a resistivity of $1 \mathrm{k} \Omega \mathrm{cm}$ than for $600 \Omega \mathrm{cm}$. This observation still fits with the manufacturer claim about the substrate resistivity which is guaranteed to be above $600 \Omega \mathrm{cm}$. The maximal extension of the depleted volume observed at $30 \mathrm{~V}$ bias is $17 \mu \mathrm{m}$. To grossly evaluate the dependence of this evaluation with the chosen reference value, we changed the reference from $z_{0}=13 \mu \mathrm{m}$ to $15 \mu \mathrm{m}$. The new estimate then becomes $20 \mu \mathrm{m}$.

\subsection{Evaluation from all of the hits}

The depth evaluation uses now ratios between the total counts of hits present in the complete distribution and not only in the full-energy peak. The reference voltage and depth are kept at the same values $\left(30 \mathrm{~V}\right.$ and $\left.z_{0}=13 \mu \mathrm{m}\right)$. Figure 8 display both experimental and TCAD estimates.

For the epitaxial layer (HR18) substrate, the maximal depth is reached at a bias voltage around $6 \mathrm{~V}$, much lower than the voltage where the depletion saturates (around $30 \mathrm{~V}$ ). This observed maximal depth is the full sensitive depth for the substrate. TCAD simulations presented in section 3 suggest that both depths are identical, which corresponds to a situation of full depletion.

Depths obtained with the bulk (CZ) substrate exceed estimates of the depleted depth by about 10 and $20 \mu \mathrm{m}$ for illuminations with energies of respectively 5.89 and $17.48 \mathrm{keV}$. This behavior 
was expected from the discussion on the electric field extension visible in figure 5. It suggests that thanks to the uniform low doping of the bulk substrate charge generated beyond the depleted volume can still be collected efficiently, even if it is spread over several pixels.

We cannot confirm robustly the reason for the difference observed between both energies. Qualitatively, the higher X-ray energy allows probing deeper the collection depth. At $17.48 \mathrm{keV}$ the weaker attenuation coefficient indeed provides higher statistics for deep photo-conversions and the larger signal guarantees a higher detection efficiency. We note that only a $15 \%$ lower detection efficiency for the photons at $5.89 \mathrm{keV}$ would suffice to explain the observed difference in estimated depths. Consequently, we assume that the full sensitive depth amounts to $35 \mu \mathrm{m}$. Using a reference depth $z_{0}$ of $15 \mu \mathrm{m}$, this depth would reach the full $40 \mu \mathrm{m}$ depth of the substrate.

\section{Conclusions and outlooks}

We have studied the depletion obtained in CMOS pixel sensors with the frontside biasing technique, where a bias voltage below $50 \mathrm{~V}$ is applied through the collecting diode. Measurements were performed on PIPPER-2, a prototype sensor which design follows the proposed frontside biasing technique. PIPPER-2 was fabricated on two high resistivity substrates: an $18 \mu \mathrm{m}$ thick thin epitaxial layer and a bulk substrate that has been thinned to $50 \mu \mathrm{m}$ ( $40 \mu \mathrm{m}$ sensitive).

Predictions for the depleted depth were obtained both with a simplified analytic approach of point-like junctions and 3D TCAD computations based on realistic sensor features and substrate profiles.

If the analytic formula is successful in predicting the slow growth of the depletion depth with the bias voltage, it fails to reproduce the dependence for high resistivities (above $1 \mathrm{k} \Omega \mathrm{cm}$ ). As a matter of fact, TCAD simulations indicate that the presence of the P-well surrounding the collecting diode limits the depletion extension. This finding correlates the maximal depleted depth with two main pixel geometry details: the P-wells extension and the pitch.

With the $22 \mu \mathrm{m}$ pixel pitch of PIPPER-2, even with a resistivity as high as $10 \mathrm{k} \Omega \mathrm{cm}$, the maximal depth of the depleted volume does not exceed $35 \mu \mathrm{m}$ within the range of applied voltages. This result suggests that the frontside biasing technique on uniformly doped substrates should be considered for depleting modest depths.

This is in fact the case for substrates featuring epitaxial layers, for which TCAD predicts full depletion up to the depth where the resistivity (hole density) stays high (low).

Experimentally, we have exploited the known attenuation coefficients of X-rays to infer depletion and collection depths from measured photon counts and one reference depth as predicted by TCAD calculus.

Our results with the PIPPER-2 sensor support that a $30 \mathrm{~V}$ bias fully depletes the epitaxial layer over $13_{-0}^{+2} \mu \mathrm{m}$. Beyond this depth, the rising hole density prevents further growth of the depletion. This configuration seems specially adapted for charged particle tracking in highly radiative environment, as already demonstrated in [12].

Study of the $600 \mathrm{k} \Omega \mathrm{cm}$ Czochralski bulk substrate indicates that the depleted volume extends to $17_{-0}^{+3} \mu \mathrm{m}$ at $40 \mathrm{~V}$ voltage bias, but that photons are still properly detected as deep as $35 \mu \mathrm{m}$. To get more detailed insights of the depleted volume and detection properties of this substrate, illumination 
with high-energy charged particles controlled with a beam telescope would be required.

Nonetheless, frontside biasing of CMOS pixel sensor fabricated on high-resistive $40 \mu \mathrm{m}$ thick bulk substrate looks a promising device for soft X-ray detection. A further paper will report on the performance of the PIPPER-2 prototype for such an application.

\section{A Analytical model}

\section{A.1 Hemispherical depleted volume from a small diode}

Here, we detail the simplified geometry describing the pixel architecture featuring small diode biased at a potential $V$. Even with simplifications, the geometry does not allow deriving easily the analytical solution for the Poisson equation. Our approximation consists in avoiding this difficulty by choosing geometries for depleted depths on both sides of the junction. It is important to note that this model intends only to estimate the depleted depths and not to reproduce details of the electric field.

The collecting diode is represented by a $n$-sides N-type prism of radius $r$ and depth $z$ sitting in a P-type substrate. The volume of the collecting diode $\left(\mathcal{V}_{\text {diode }}\right)$ and the junction area $(\mathcal{A})$ can be computed using the parameters $n, r$ and $z$.

$$
\mathcal{V}_{\text {diode }}=n z r^{2} \tan \frac{\pi}{n}, \quad \mathcal{A}=n r \tan \frac{\pi}{n}(r+2 z) .
$$

On each side of the junction, the charge in a depleted volume $\mathcal{V}$ is

$$
Q=q N_{e f f} \mathcal{V} .
$$

Inside the N-type prism $\left(N_{e f f}=N_{D}\right)$, with $W_{N}$ the depletion width over the contact area of the junction area, the depleted volume is

$$
\mathcal{V}_{N}=\mathcal{A} W_{N} .
$$

The width of the depleted volume on this side of the junction is taken from the 1D planar case [13] and is expressed by

$$
W_{N}=\sqrt{\frac{2 \epsilon_{S i}}{q} \frac{N_{A}}{N_{D}}\left(\frac{1}{N_{A}+N_{D}}\right)\left(V_{b i}+|V|\right)} .
$$

This choice is driven by the quite flat aspect of the collecting diode, which is indeed much wider than thick. We crosschecked that the typical widths obtained within the conditions discussed in this paper represent a depth 5 to 6 orders of magnitude lower than the diode thickness.

Another possible ansatz would have been to consider a semi-spherical diode. In that case the depletion depth $W_{N}$ changes with the cubic root of the bias voltage and could reach 10 or 100 times the depth predicted by the planar ansatz. However, using this alternative geometry leads to depletion depths of the sensitive volume, $W_{P}$, only 25 to $30 \%$ higher. The finite element simulations and experimental results tend to favour the predictions with the planar ansatz, which set our final choice a posteriori. 
Thus, the charge on the n-side of the junction is

$$
Q_{N}=\sqrt{2 \epsilon_{S i} q\left(\frac{N_{A} N_{D}}{N_{A}+N_{D}}\right) \mathcal{A}^{2}\left(V_{b i}+|V|\right)} .
$$

With the small N-type diode sitting in the P-type substrate, one can assume a hemispherical expansion of the depleted volume. This depleted volume can be expressed as

$$
\mathcal{V}_{P}=\frac{2}{3} \pi W_{P}^{3}-\mathcal{V}_{\text {diode }}
$$

The charge in the depleted volume on the P-side $\left(N_{\text {eff }}=N_{A}\right)$ is

$$
Q_{P}=q N_{A}\left(\frac{2}{3} \pi W_{P}^{3}-\mathcal{V}_{\text {diode }}\right)
$$

To respect the neutrality of the semiconductor, the charge on each side of the junction is equal $\left(Q_{P}=Q_{N}\right)$. By considering an asymmetric junction with a highly doped $\mathrm{N}$-side and a low doped P-side $\left(N_{D}>>N_{A}\right)$, the radius of the hemispherical expansion can finally be computed as:

$$
W_{P}=\sqrt[3]{\frac{3}{2 \pi}\left(\sqrt{\frac{2 \epsilon_{S i}}{q N_{A}} \mathcal{A}^{2}\left(V_{b i}+|V|\right)}+\mathcal{V}_{\text {diode }}\right)} .
$$

\section{A.2 Approximation for merged depleted volumes}

When merging occurs between the depleted volumes of adjacent pixels, the charge neutrality within a single pixel should take into account that part of the volume initially attributed to this pixel is "merged" in neighbour pixels. The merged volume can be estimated as two spherical caps (four halves). With $h=W_{P}-s / 2$ the height and $a=\sqrt{h\left(2 W_{P}-h\right)}$ the radius of the base of the cap [14], the merged volume can be expressed as

$$
\begin{aligned}
& \mathcal{V}_{\text {merged }}=\frac{2}{3} \pi h^{2}\left(3 W_{P}-h\right), \\
& \mathcal{V}_{\text {merged }}=\frac{2}{3} \pi\left(W_{P}-\frac{s}{2}\right)^{2}\left(2 W_{P}+s / 2\right) .
\end{aligned}
$$

Since in this situation the radius of the hemisphere is larger than the pitch, the bottom of the depleted zone can grossly be considered flat. So the merged volume is simply added to the truncated hemisphere as a cuboid of volume $s \times s \times W_{\text {merging. }}$. The added width is thus given by

$$
W_{\text {merging }}=\frac{\mathcal{V}_{\text {merged }}}{s^{2}} .
$$

The total width then become:

$$
\begin{aligned}
& W_{P_{\text {tot }}}=W_{P}+W_{\text {merging }}, \\
& W_{P_{\text {tot }}}=W_{P}+\frac{\frac{2}{3} \pi\left(W_{P}-\frac{s}{2}\right)^{2}\left(2 W_{P}+s / 2\right)}{s^{2}} .
\end{aligned}
$$




\section{References}

[1] Giacomo Contin, Leo Greiner, Joachim Schambach, Michal Szelezniak, Eric Anderssen, Jacque Bell, Mario Cepeda, Thomas Johnson, Hao Qiu, Hans-Georg Ritter, Joseph Silber, Thorsten Stezelberger, Xiangming Sun, Co Tran, Chinh Vu, Howard Wieman, Kenneth Wilson, Rhonda Witharm, Samuel Woodmansee, and John Wolf. The STAR MAPS-based PiXeL detector. Nuclear Instruments and Methods in Physics Research Section A: Accelerators, Spectrometers, Detectors and Associated Equipment, 2018.

[2] W. Snoeys. Monolithic CMOS sensors for high energy physics. Nuclear Instruments and Methods in Physics Research Section A: Accelerators, Spectrometers, Detectors and Associated Equipment, 2018.

[3] R. Turchetta, P.P. Allport, G. Casse, A. Clark, J. Crooks, A. Evans, A. Fant, A.R. Faruqi, M.J. French, R. Henderson, Q. Morrissey, D. Prior, M. Prydderch, J.J. Velthuis, G. Villani, N. Waltham,

B. Willmore, and P. Willmore. CMOS Monolithic Active Pixel Sensors (MAPS): New 'eyes' for science. Nuclear Instruments and Methods in Physics Research Section A: Accelerators, Spectrometers, Detectors and Associated Equipment, 560(1):139 - 142, 2006. Proceedings of the 13th International Workshop on Vertex Detectors.

[4] Takaki Hatsui and Heinz Graafsma. X-ray imaging detectors for synchrotron and XFEL sources. IUCrJ, 2(3):371-383, May 2015.

[5] Gianluca Aglieri Rinella. The ALPIDE pixel sensor chip for the upgrade of the ALICE Inner Tracking System. Nuclear Instruments and Methods in Physics Research Section A: Accelerators, Spectrometers, Detectors and Associated Equipment, 845:583 - 587, 2017. Proceedings of the Vienna Conference on Instrumentation 2016.

[6] T. Wang, M. Barbero, I. Berdalovic, C. Bespin, S. Bhat, P. Breugnon, I. Caicedo, R. Cardella, Z. Chen, Y. Degerli, N. Egidos, S. Godiot, F. Guilloux, T. Hemperek, T. Hirono, H. Kruger, T. Kugathasan, F. Hugging, C. A. Marin Tobon, K. Moustakas, P. Pangaud, P. Schwemling, H. Pernegger, D-L. Pohl, A. Rozanov, P. Rymaszewski, W. Snoeys, and N. Wermes. Depleted fully monolithic CMOS pixel detectors using a column based readout architecture for the ATLAS Inner Tracker upgrade, 2017.

[7] Niloufar Alipour Tehrani. Recent results with HV-CMOS and planar sensors for the CLIC vertex detector. Nuclear Instruments and Methods in Physics Research Section A: Accelerators, Spectrometers, Detectors and Associated Equipment, 845:1 - 7, 2017. Proceedings of the Vienna Conference on Instrumentation 2016.

[8] V Vervisch, Y Larmande, P Delaporte, T Sarnet, M Sentis, H Etienne, F Torregrosa, Cristiano F, and P. F. Fazzini. Laser activation of Ultra Shallow Junctions (USJ) doped by Plasma Immersion Ion Implantation (PIII). Applied Surface Science, 255(10):5647 - 5650, 2009. Laser and Plasma in Micro- and Nano-Scale Materials Processing and Diagnostics.

[9] S. M. Sze. Semiconductor Devices. Physics and Technology. John Wiley \& Sons, Inc., 1985.

[10] J. B. Lincelles. Étude d'imageurs CMOS fortement dépeuplés pour l'amélioration des performances des futurs instruments d'observation spatiaux. PhD thesis, École Doctorale Génie Électrique, Électronique et Télécommunications (Toulouse), 2015.

[11] NIST. Physical Reference Data.

[12] Alejandro Perez Perez. Radiation tolerance enhancement of high resolution cmos pixel sensors. In Presented at IEEE-NSS conference, 29/10-6/11, Strasbourg, 2016.

[13] S. M. Sze. Physics of semiconductor devices, Third Edition. John Wiley \& Sons, Inc., 1985. 
[14] Polyanin A. D. and Manzhirov A. V. Handbook of Mathematics for Engineers and Scientists. Chapman and Hall/CRC, 2006. 